\title{
Acné y lácteos: ¿existe una asociación?
}

\section{Acne and dairy: Is there an association?}

\author{
Karla Leticia Valdés-Morales, ${ }^{*}$ Ana Karen Romero-Guzmán,, \\ María Teresa Rosas-Morett, ${ }^{\S}$ Martha Alejandra Morales-Sánchez ${ }^{\llbracket}$
}

\section{RESUMEN}

Históricamente, la relación entre el acné y la dieta ha sido controversial. En los últimos años, se han estudiado diversos factores dietéticos y su relación con el acné; de los más estudiados han sido los lácteos. Se han propuesto diversos componentes en la leche que pueden estar involucrados en la fisiopatogenia del acné, tanto directa como indirectamente. Además, se han realizado múltiples estudios observacionales en diferentes poblaciones en los que se intenta corroborar o descartar esta asociación; dichos estudios culminaron en la publicación de tres revisiones sistemáticas con metaanálisis. En esta revisión se exponen los puntos más importantes encontrados en la literatura en cuanto a acné y lácteos.

Palabras clave: Acné, dieta, lácteos.

\section{ABSTRACT}

Historically, relationship between acne and diet has been controversial. Over the last few years, different foods and nutrition factors and their relationship with acne have been studied; dairy products have been some of the most studied. Several components of dairy products have been implicated in acne pathogenesis, with direct and indirect mechanisms. Multiple observational studies trying to determine the relationship of dairy and acne have been done on different populations; these studies led to the publication of three systematic reviews and metaanalysis. On this review, we cover the most important concepts regarding dairy products and acne found in literature.

Keywords: Acne, diet, dairy.

\section{INTRODUCCIÓN}

El acné es una enfermedad muy común y es uno de los principales motivos de consulta dermatológica; se ha reportado que afecta hasta $95 \%$ de adolescentes hombres y $85 \%$ de adolescentes mujeres, de éstos hasta $20 \%$ presentará acné moderado a severo y en una gran mayoría persistirá hasta la adultez..$^{1-3}$ Clínicamente esta entidad se caracteriza por la presencia de comedones, pápulas y pústulas. ${ }^{4}$ Se han estudiado diversos factores ambientales asociados al acné; su relación con la dieta data de manera formal desde

\footnotetext{
* Residente de tercer año de Dermatología.

₹ Residente de segundo año de Dermatología.

$\S$ Residente de primer año de Dermatología.

" Jefe de Enseñanza e Investigación.

Centro Dermatológico «Dr. Ladislao de la Pascua», SSCDMX.
}

1885 cuando Bulkley publicó estudios dietéticos en 1,500 pacientes con acné. Posteriormente en 1949, Robinson sugirió a su vez el probable rol de los lácteos en la patogenia del acné. ${ }^{5,6}$

Por otra parte, también se encuentra la percepción de los pacientes de la dieta con el acné; en una encuesta realizada a 49 casos, $92 \%$ percibía que el acné se asociaba con la dieta, y de éstos $47 \%$ percibía asociación entre el acné y los lácteos. ${ }^{7}$

Aún existe controversia entre dicha asociación, hasta el momento se han realizado estudios observacionales y revisiones sistemáticas que han tratado de encontrar relación. Es importante conocer las bases científicas acerca de la dieta con el acné y establecer su asociación para poder realizar recomendaciones adecuadas. $^{8}$

Citar como: Valdés-Morales KL, Romero-Guzmán AK, Rosas-Morett MT, MoralesSánchez MA. Acné y lácteos: ¿existe una asociación? Rev Cent Dermatol Pascua. 2020; 29 (2-3): 56-60. https://dx.doi.org/10.35366/99917 


\section{¿CÓMO AFECTAN LOS LÁCTEOS EL ACNÉ?}

Para comprender la asociación entre los lácteos y el acné es necesario abordar su biopatología; el acné es una enfermedad crónica inflamatoria que afecta a la unidad pilosebácea y se asocia con hiperseborrea, alteración en los ácidos grasos, desregulación hormonal, hiperqueratinización folicular e inducción de la inflamación por medio del sistema inmune. ${ }^{4}$

El incremento de los niveles de factor de crecimiento similar a insulina (IGF-1) se ha correlacionado con mayor número de lesiones de acné ya que promueve la proliferación de queratinocitos e incrementa la síntesis lipídica, ${ }^{4}$ los aminoácidos derivados de la leche y la caseína se han asociado a un incremento del mismo. .-11 $^{-11}$ El incremento en los niveles de IGF-1 activa el factor de transcripción Forkhead-box-O1 (FoxO1) que es responsable de la regulación de la secreción de glándulas sebáceas, así como un modulador del complejo 1 de la diana de rapamicina en células de mamífero (mTORC1), molécula que se ha asociado a enfermedades inflamatorias como la obesidad y la diabetes, y que en estudios recientes se ha implicado en la activación de sebocitos y acneogénesis. ${ }^{12-14}$ Por otra parte, IGF-1 incrementa los niveles de receptores de testosterona y andrógenos, lo que culmina en formación de lesiones de acné, ${ }^{15,16}$ asimismo, la leche contiene andrógenos y factores de crecimiento que a su vez pueden aumentar los niveles de IGF-1. Debe enfatizarse que no es el contenido de IGF-1 el responsable de estos eventos, sino la estimulación provocada por aminoácidos en la leche que promueve la síntesis de IGF-1. ${ }^{17}$

Las proteínas de la leche tienen un rol en el desarrollo del acné, los aminoácidos esenciales de cadena ramificada derivados de la leche pueden inducir secreción de insulina postprandial. Se ha demostrado que la leche causa un incremento desproporcionado de los niveles de insulina, con una respuesta insulinémica hasta seis veces mayor a lo correspondiente a su índice glucémico. ${ }^{15,18}$ La lactoalbúmina, otro componente de la leche, puede estimular la producción de IGF-1 y activar la vía mTORC1. Por el otro lado, algunos estudios se han centrado en la betacelulina, proteína de la leche que se encarga de activar múltiples factores de crecimiento como el factor de crecimiento epidérmico expresado en queratinocitos y sebocitos, resultando en la activación de la fosfoinositol-3-cinasa (PI3K) y en la proliferación de los mismos. ${ }^{19}$

Algunos estudios confirman que la leche pasteurizada contiene microRNA bioactivos que interfieren directamente con la activación de IGF-1. ${ }^{17}$ A su vez el
microRNA-148a suprime la DNA-metiltransferasa-1, la cual promueve señalización androgénica incrementando el riesgo de acné. ${ }^{20}$ Se ha determinado que la ebullición y fermentación disuelven estas moléculas, por lo que su relación con el desarrollo de lesiones de acné se ve disminuido en productos derivados de la leche, como se mencionará más adelante. ${ }^{11,20}$

A continuación, desglosaremos algunos de los estudios más representativos que se han realizado para evaluar la relación entre el consumo de lácteos y el acné, incluyendo los tres metaanálisis.

\section{ASOCIACIÓN ENTRE LÁCTEOS Y ACNÉ}

Se han realizado múltiples estudios observacionales, en los cuales se valora la asociación entre lácteos y acné. En 2018, se publicaron tres revisiones sistemáticas con metaanálisis. Los estudios incluidos fueron similares, mas no idénticos por diferentes criterios de selección y distintos análisis por subgrupo. Aghasi y colaboradores incluyeron 14 estudios en los que se analizaron cuatro de cohorte, siete casos y controles y tres transversales; el rango de edad fue de 9 a 30 años. ${ }^{11}$ Juhl y su grupo incluyeron 14 estudios, de los cuales cuatro fueron longitudinales, cinco de casos y controles y cinco de transversales, que incluían pacientes de siete a 30 años de edad. ${ }^{21}$ Por último, Dai y su equipo incluyeron 13 estudios, de los cuales cuatro eran de cohorte, cuatro casos y controles y cinco transversales, con rango de edad de nueve a 60 años. ${ }^{17}$ En los análisis de Aghasi y Juhl se incluyó la asociación entre la ingesta de cualquier lácteo con el acné. Aghasi y colaboradores incluyeron cinco estudios con asociación estadísticamente significativa (Odds Ratio (OR) 2.16, IC 95\% 1.20-5.67); sin embargo, se reportó heterogeneidad importante entre estudios; Juhl y su equipo reportaron un OR combinado de 1.25 (IC 95\% 1.15-1.36) al combinar el OR de todos los estudios incluidos.

En un subgrupo de tres estudios, Dai ${ }^{17}$ y colaboradores reportaron una asociación no significativa entre consumo de leche y acné leve (OR 1.14, IC 95\% 0.861.51); sin embargo, en el subgrupo de cinco estudios en los que se incluyeron casos de acné moderado-grave sí se encontró una asociación estadísticamente significativa (OR 1.18, IC 95\% 1.01-1.37).

\section{TIPOS DE LECHE Y ACNÉ}

Comúnmente la leche de vaca está disponible en diferentes presentaciones de acuerdo al contenido de grasa: leche entera contiene $3.5 \%$ de grasa; leche baja 
en grasa contiene $2 \%$ de grasa; y sin grasa o leche descremada. Adebamowo y colaboradores en 2006 investigaron la relación entre lácteos y acné en más de 47,000 mujeres adultas a las que se les realizó un cuestionario con respecto a su dieta durante la preparatoria. Encontraron que la leche descremada se correlacionaba significativamente con acné OR 1.44 (IC 95\% 1.21-1.72; $\mathrm{p}=0.003$ ) comparado con la leche entera OR 1.12 (IC $95 \% 1-1.25 ; p=0.56) .{ }^{22}$ Este estudio fue muy criticado por su diseño retrospectivo, por lo que el mismo grupo de investigación realizó otros dos estudios en 273 niños y 94 niñas adolescentes (9 a 15 años) confirmando los resultados previos, en ambos fue significativo el consumo de leche descremada con un OR de 1.19..23,24

Por el contrario, LaRosa y su grupo condujeron en 2016 un estudio de casos y controles en 225 pacientes con edad entre 14-19 años, reportando que la leche descremada desencadena acné más que la leche entera, con diferencia estadísticamente significativa entre el consumo de leche diario de pacientes con acné que aquéllos sin acné $(p=0.01)$. La limitante del estudio es que incluyó cuestionarios de autorreporte en cuanto a la ingesta. ${ }^{25}$ Igualmente, en un estudio longitudinal de adolescentes noruegos ( $n=2,489$ ), no se encontró correlación entre acné y la ingesta de leche, yogur y queso. ${ }^{26}$

Los tres metaanálisis demostraron una asociación entre el consumo de leche y el acné como se muestra en la Tabla 1. En el estudio de Aghasi y colaboradores se calculó la OR para cada tipo de leche: leche entera OR 1.48 (IC 95\% 1.31-1.66), leche baja en grasa OR 1.25 (IC 95\% 1.10-1.43) y leche descremada OR 1.82 (IC 95\% 1.34-2.47). ${ }^{11}$ Los resultados del metaanálisis de Juhl fueron: OR 1.22 (IC 95\% 1.06-1.41) para leche entera y OR 1.32 (IC 95\% 1.16-1.52) para leche baja en grasa/descremada. ${ }^{21}$ Por último, Dai y su grupo calcularon: leche entera OR 1.13 (IC 95\% 1.05-1.21), leche baja en grasa OR 1.14 (IC 95\% 1.08-1.22) y le- che descremada OR 1.24 (IC 95\% 1.13-1.37). ${ }^{17}$ En los tres estudios se demuestra una mayor asociación del acné con el consumo de leche descremada. Se han postulado dos hipótesis acerca de estos resultados: la leche entera contiene mayor cantidad de grasa, por lo que produce mayor saciedad con menor ingesta, mientras que la leche con menor porcentaje de grasa requiere de mayor ingesta para la saciedad, llevando a un mayor consumo de proteína de leche ${ }^{17}$ Asimismo, se ha hipotetizado que en el procesamiento de la leche baja en grasa y descremada puede disminuir la concentración de moléculas responsables de la producción de acné. ${ }^{11}$

Después de la publicación del metaanálisis, Juhl y colaboradores realizaron un estudio con aleatorización mendeliana en 20,416 adultos daneses (basados en el alelo asociado a producción de lactasa) para corroborar los hallazgos en su estudio; sin embargo, en su población no se encontró una relación estadísticamente significativa entre acné y el consumo de leche. ${ }^{27}$ Por otro lado, en 2019 se publicó un nuevo estudio de casos y controles realizado en Kabul con 279 casos de 10-24 años, en donde se demostró una asociación menor con leche baja en grasa que con leche entera OR 1.95 (IC $95 \% 1.10-3.45) .^{5}$

\section{CANTIDAD DE CONSUMO DE LECHE Y ACNÉ}

Además del tipo de leche, también se ha analizado si un consumo alto se relaciona con un mayor riesgo para acné, encontrando resultados muy variables.

El estudio realizado en Kabul mostró que el consumo de leche entera más de 3 días por semana se asoció a acné moderado o severo OR 2.36 (IC 95\% 1.39-4.01). ${ }^{5}$ En Malasia, un estudio de casos y controles (57 casos y 57 controles) demostró que el consumo de dos vasos de leche al día incrementaba la incidencia de acné hasta el doble. ${ }^{28}$ En el estudio de Ulvestad y colaboradores se encontró que la incidencia de acné era más alta en

\begin{tabular}{|c|c|c|c|}
\hline & $\begin{array}{c}\text { Dai }^{17} \\
\text { OR (IC95\%) }\end{array}$ & $\begin{array}{c}\text { Aghasi }^{11} \\
\text { OR (IC95\%) }\end{array}$ & $\begin{array}{c}\text { Juhl }^{21} \\
\text { OR }(\mathrm{IC} 95 \%)\end{array}$ \\
\hline Cualquier leche & $1.16(1.09-1.24)$ & $1.48(1.31-1.66)$ & $1.28(1.13-1.44)$ \\
\hline Leche entera & $1.13(1.05-1.21)$ & Sin asociación 1.48 (1.31-1.66) & $1.22(1.06-1.41)$ \\
\hline
\end{tabular}

$\mathrm{OR}=$ odds ratio; $\mathrm{IC}$ = intervalo de confianza. 
hombres y mujeres que consumían dos o más tazas de leche entera al día. ${ }^{26} \mathrm{Di}$ Landro y su equipo condujeron un estudio de casos-controles en Italia, incluyendo 205 pacientes de 10 a 24 años con acné moderado-severo y 358 controles sin acné o acné leve; reportaron un riesgo incrementado OR 1.78 (IC 95\% 2.31-5.05) en los que consumían más de tres porciones de leche a la semana. ${ }^{29}$ En Nigeria, se llevó a cabo un estudio transversal en adolescentes y la prevalencia de acné fue mayor en quienes reportaron la ingesta diaria de leche como bebida (72.6 vs $62.0 \%) .{ }^{30}$

Por otra parte, los tres metaanálisis coinciden en la relación entre el consumo de leche y el acné. Juhl y colaboradores encontraron una relación proporcional entre mayor consumo y acné: un consumo de dos o más vasos al día con un OR de 1.43 (IC 95\% 1.091.88), un vaso por día con un OR de 1.41 (IC 95\% 1.05-1.90); el consumo de dos a seis vasos por semana sin asociación estadísticamente significativa (OR 1.24, IC 95\% 0.95-1.62). ${ }^{21}$ Dai y su grupo reportaron que el consumo de un vaso de leche al día o menos se asociaba a acné con un OR de 1.08 (IC 95\% 1.001.17) y el consumo mayor a un vaso por día con un OR de1.12 (IC 95\% 1.01-1.24). ${ }^{17}$ El grupo de Aghasi y colaboradores encontró un mayor riesgo para acné en los pacientes con mayor consumo de leche, a diferencia de los de menor consumo OR 1.48 (IC 95\% 1.31-1.66), dicho riesgo se incrementaba 83,13 y $26 \%$ por cada ración diaria adicional de lácteos, leche entera y leche descremada respectivamente. ${ }^{11}$

\section{OTROS LÁCTEOS}

Hasta el momento, no se ha encontrado una relación significativa entre la ingesta de queso, yogur y acné. Aghasi y colaboradores incluyeron cuatro estudios en su metaanálisis sin encontrar asociación entre el consumo de yogur/queso y acné (OR 0.90, IC 95\% 0.73-1.11). ${ }^{11}$ El grupo de Juhl reportó un OR de 1.22 (IC 95\% 1.0-1.5, $\mathrm{p}=0.05$ ) para la ingesta de queso y un OR de 1.36 (IC $95 \% 1.05-1.77, p=0.02$ ) para la ingesta de yogur. ${ }^{21}$

\section{CONCLUSIÓN}

La evidencia actual señala una relación positiva entre el consumo de leche y acné; sin embargo, está basada principalmente en estudios observacionales con un nivel de calidad bajo-moderado. En las tres revisiones sistemáticas existentes, los autores concuerdan en lo siguiente: la heterogeneidad de los estudios incluidos y el número limitado de investigaciones disponibles para cada análisis de subgrupo. Además, en algunos de los estudios incluidos, el diagnóstico de acné era reportado por el mismo paciente en cuestionarios ${ }^{22-24,26,31-33}$ y para valorar el consumo de lácteos utilizaron diferentes cuestionarios dietéticos.

Hasta la fecha, no hay ningún estudio aleatorizado de alta calidad para hacer una fuerte recomendación en cuanto a disminuir el consumo. Sin embargo, diseñar un estudio prospectivo o incluso un ensayo clínico para determinar la asociación entre lácteos y acné es difícil. A pesar de las debilidades reportadas en los estudios observacionales, todos demuestran una tendencia positiva hacia esta asociación y es la evidencia con la que contamos.

Con todo esto, podemos advertir que el consumo de leche (entera, baja en grasa y descremada) puede estar asociado a un incremento en el acné; mientras que los derivados (yogur y queso) no han demostrado dicha relación. Por lo tanto, recomendar disminuir el consumo de leche, en particular leche descremada, posee un grado de recomendación moderado y debe ser individualizado a cada paciente.

Por último, el pilar del tratamiento para el acné no lo constituye la dieta, es sólo uno de los muchos factores involucrados en la etiopatogenia de la enfermedad. Es importante que este concepto lo transmitamos a nuestros pacientes para el mejor entendimiento de su dermatosis y evitar que se basen en recomendaciones externas poco fundamentadas.

\section{REFERENCIAS}

1. Burton JL, Cunliffe WJ, Stafford I, Shuster S. The prevalence of acne vulgaris in adolescence. Br J Dermatol. 1971; 85: 119-126.

2. Ghodsi SZ, Orawa H, Zouboulis CC. Prevalence, severity, and severity risk factors of acne in high school pupils: a community-based study. J Invest Dermatol. 2009; 129: 2136-2141.

3. Thiboutot D, Gollnick H, Bettoli V, Dréno B, Kang S, Leyden JJ et al. New insights into the management of acne: an update from the global alliance to improve outcomes in acne group. J Am Acad Dermatol. 2009; 60: S1-50.

4. Moradi TS, Makrantonaki E, Ganceviciene R, Dessinioti C, Feldman SR, Zouboulis CC. Acne vulgaris. Nat Rev Dis Primers. 2015; 1: 15029.

5. Aalemi AK, Anwar I, Chen H. Dairy consumption and acne: a case control study in Kabul, Afghanistan. Clin Cosmet Investig Dermatol. 2019; 12: 481-487.

6. Robinson HM. The acne problem. South Med J. 1949; 42: 1050-1060, illust.

7. Nguyen QG, Markus R, Katta R. Diet and acne: an exploratory survey study of patient beliefs. Dermatol Pract Concept. 2016; 6: 21-27.

8. Bronsnick T, Murzaku EC, Rao BK. Diet in dermatology: part I. Atopic dermatitis, acne, and nonmelanoma skin cancer. J Am Acad Dermatol. 2014; 71: 1039.e1-1039.e12. 
9. Rich-Edwards JW, Ganmaa D, Pollak MN, Nakamoto EK, Kleinman $\mathrm{K}$, Tserendolgor $\mathrm{U}$ et al. Milk consumption and the prepubertal somatotropic axis. Nutr J. 2007; 6: 28.

10. Rahaman SMA, De D, Handa S, Pal A, Sachdeva N, Ghosh T et al. Association of insulin-like growth factor (IGF)-1 gene polymorphisms with plasma levels of IGF-1 and acne severity. J Am Acad Dermatol. 2016; 75: 768-773.

11. Aghasi M, Golzarand M, Shab-Bidar S, Aminianfar A, Omidian M, Taheri F. Dairy intake and acne development: a meta-analysis of observational studies. Clin Nutr. 2019; 38: 1067-1075.

12. Agamia NF, Abdallah DM, Sorour O, Mourad B, Younan DN. Skin expression of mammalian target of rapamycin and forkhead box transcription factor 01, and serum insulin-like growth factor-1 in patients with acne vulgaris and their relationship with diet. $\mathrm{Br} \mathrm{J}$ Dermatol. 2016; 174: 1299-1307.

13. Cerman AA, Aktas E, Altunay I, Arici JE, Tulunay A, Ozturk FY. Dietary glycemic factors, insulin resistance, and adiponectin levels in acne vulgaris. J Am Acad Dermatol. 2016; 75: 155-162.

14. Melnik BC. Acne vulgaris: the metabolic syndrome of the pilosebaceous follicle. Clin Dermatol. 2018; 36: 29-40.

15. Melnik BC. Linking diet to acne metabolomics, inflammation, and comedogenesis: an update. Clin Cosmet Investig Dermatol. 2015; 8: 371-388.

16. Danby FW. Diet and acne. Clin Dermatol. 2008; 26: 93-96.

17. Dai R, Hua W, Chen W, Xiong L, Li L. The effect of milk consumption on acne: a meta-analysis of observational studies. J Eur Acad Dermatol Venereol. 2018; 32: 2244-2253.

18. Ostman EM, Liljeberg Elmstahl HG, Bjorck IM. Inconsistency between glycemic and insulinemic responses to regular and fermented milk products. Am J Clin Nutr. 2001; 74: 96-100.

19. Melnik BC. Evidence for acne-promoting effects of milk and other insulinotropic dairy products. Nestle Nutr Workshop Ser Pediatr Program. 2011; 67: 131-145.

20. Melnik BC. Milk disrupts p53 and DNMT1, the guardians of the genome: implications for acne vulgaris and prostate cancer. Nutr Metab (Lond). 2017; 14: 55.

21. Juhl CR, Bergholdt HKM, Miller IM, Jemec GBE, Kanters JK, Ellervik C. Dairy intake and acne vulgaris: a systematic review and metaanalysis of 78,529 children, adolescents, and young adults. Nutrients. 2018; 10: 1049.

22. Adebamowo CA, Spiegelman D, Danby FW, Frazier AL, Willett WC, Holmes MD. High school dietary dairy intake and teenage acne. $J$ Am Acad Dermatol. 2005; 52: 207-214.
23. Adebamowo CA, Spiegelman D, Berkey CS, Danby FW, Rockett $\mathrm{HH}$, Colditz GA et al. Milk consumption and acne in adolescent girls. Dermatol Online J. 2006; $12: 1$.

24. Adebamowo CA, Spiegelman D, Berkey CS, Danby FW, Rockett HH, Colditz GA et al. Milk consumption and acne in teenaged boys. $J \mathrm{Am}$ Acad Dermatol. 2008; 58: 787-793.

25. LaRosa CL, Quach KA, Koons K, Kunselman AR, Zhu J, Thiboutot $D M$ et al. Consumption of dairy in teenagers with and without acne. J Am Acad Dermatol. 2016; 75: 318-322.

26. Ulvestad M, Bjertness E, Dalgard F, Halvorsen JA. Acne and dairy products in adolescence: results from a Norwegian longitudinal study. J Eur Acad Dermatol Venereol. 2017; 31: 530-535.

27. Juhl CR, Bergholdt HKM, Miller IM, Jemec GBE, Kanters JK, Ellervik C. Lactase persistence, milk intake, and adult acne: a mendelian randomization study of 20,416 danish adults. Nutrients. 2018; 10: 1041.

28. Suppiah TSS, Sundram TKM, Tan ESS, Lee CK, Bustami NA, Tan CK. Acne vulgaris and its association with dietary intake: a Malaysian perspective. Asia Pac J Clin Nutr. 2018; 27: 1141-1145.

29. Di Landro A, Cazzaniga S, Parazzini F, Ingordo V, Cusano F, Atzori $L$ et al. Family history, body mass index, selected dietary factors, menstrual history, and risk of moderate to severe acne in adolescents and young adults. J Am Acad Dermatol. 2012; 67: 1129-1135.

30. Okoro EO, Ogunbiyi AO, George AO, Subulade MO. Association of diet with acne vulgaris among adolescents in Ibadan, southwest Nigeria. Int J Dermatol. 2016; 55: 982-988.

31. Tsoy N. The influence of dietary habits on acne. World J Med Sci. 2013; 8: 212-216.

32. Wolkenstein P, Misery L, Amici JM, Maghia R, Branchoux S, Cazeau C et al. Smoking and dietary factors associated with moderate-to-severe acne in French adolescents and young adults: results of a survey using a representative sample. Dermatology. 2015; 230: 34-39.

33. Wolkenstein P, Machovcova A, Szepietowski JC, Tennstedt D, Veraldi $S$, Delarue A. Acne prevalence and associations with lifestyle: a crosssectional online survey of adolescents/young adults in 7 European countries. J Eur Acad Dermatol Venereol. 2018; 32: 298-306.

\section{Correspondencia:}

Dra. Martha Alejandra Morales Sánchez

Dr. Vértiz Núm. 464, Esq. Eje 3 Sur,

Col. Buenos Aires, Alcaldía Cuauhtémoc, 06780, Ciudad de México, México

Tel.: 5538-7033

E-mail: marthamoralessanchez@gmail.com 